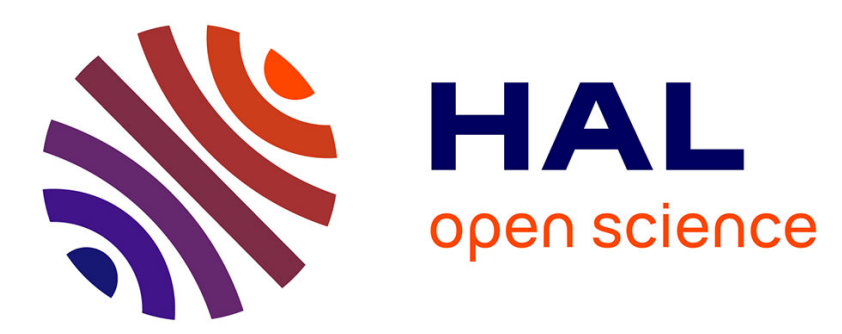

\title{
Restricted dissolution and derivatization capacities of cellulose fibres under uniaxial elongational stress
}

Nicolas Le Moigne, Monica Spinu, Thomas Heinze, Patrick Navard

\section{To cite this version:}

Nicolas Le Moigne, Monica Spinu, Thomas Heinze, Patrick Navard. Restricted dissolution and derivatization capacities of cellulose fibres under uniaxial elongational stress. Polymer, 2010, 51 (2), pp.Pages 447-453. 10.1016/j.polymer.2009.11.053 . hal-00509587

\section{HAL Id: hal-00509587}

https://hal-mines-paristech.archives-ouvertes.fr/hal-00509587

Submitted on 2 May 2011

HAL is a multi-disciplinary open access archive for the deposit and dissemination of scientific research documents, whether they are published or not. The documents may come from teaching and research institutions in France or abroad, or from public or private research centers.
L'archive ouverte pluridisciplinaire HAL, est destinée au dépôt et à la diffusion de documents scientifiques de niveau recherche, publiés ou non, émanant des établissements d'enseignement et de recherche français ou étrangers, des laboratoires publics ou privés. 


\title{
Restricted dissolution and derivatization capacities of cellulose fibres under uniaxial elongational stress
}

\author{
Nicolas Le Moigne ${ }^{1}$, Monica Spinu ${ }^{1,2}$, Thomas Heinze ${ }^{2}$ and Patrick Navard ${ }^{1}$
}

1- Mines ParisTech, CEMEF - Centre de Mise en Forme des Matériaux, CNRS UMR 7635, BP 207, 1 Rue Claude Daunesse, F-06904 Sophia Antipolis Cedex, France

2- Kompetenzzentrum Polysaccharidforschung, Friedrich-Schiller-Universität Jena, Humboldtstrasse 10, D-07743 Jena, Germany

Members of EPNOE, the European Polysaccharide Network of Excellence: www.epnoe.eu

* Corresponding author. Tel: 33(0)493957466. Fax: 33(0)492389752. E-mail: patrick.navard@minesparistech.fr 


\begin{abstract}
Cellulose is a future major source of materials and biofuel but its extraction and its chemical or enzymatic treatments are difficult, polluting and inefficient tasks. The accessibility of the reagents to cellulose chains is indeed limited. Classical evocated reasons for this lack of accessibility are pore structure, tight hydrogen bond arrays, crystallinity and presence of resistant materials like lignin. Studying dissolution of cotton hairs and regenerated cellulose fibres in various solvents under uniaxial tension, we found that tension is preventing these fibres to dissolve in chemicals that would dissolve the same cellulose fibres tension-free. We show that what is controlling dissolution is not the degree of swelling since, at the same degree of swelling, fibres under tension does not dissolve while fibres without tension do. An important result is that when a fibre under tension (thus swollen but not dissolved) is breaking, it is immediately dissolving. Under tension, when the solvent is present around cellulose chains, it is activated to solvate the chains only when tension stress is released. A chemical reaction like acetylation of cellulose fibre under tension also gives an interesting result. The degree of substitution remains very low while the same experiment performed without tension leads to higher degree of substitution followed by the dissolution of the fibre (even increasing further the DS due to homogeneous reaction). We postulate that the lack of dissolution capacity or reacting activity under tension can be due to the hampering of local conformational movements, the cellulose chains being not able to perform axial movements. The availability of performing local conformational movements could be a main component of cellulose activation.
\end{abstract}

Keywords: cellulose, fibre, dissolution, reactivity, tension 


\section{Introduction}

It is well known that cellulose present in cell walls is not "very accessible" [1], an empirical statement reflecting the difficulty to extract or treat cellulose. To overcome this accessibility problem, cell walls are usually swollen in various chemicals and/or under different thermodynamic conditions (like steam explosion) before cellulose can be extracted or treated. Classical evoked reasons for nonaccessibility or difficulties to be treated are like limited pore accessibility, high chain length, high crystallinity, presence of hydrogen bonds or influence of other molecules like hemicelluloses or lignin [2]. It has been suggested that these reasons are not giving the full story and that other "long range" interactions should be hypothesized, without being able to identify the nature of these interactions [3].

During the course of a study on cellulose dissolution, we found incidentally that imposing a small uniaxial tension on cellulose fibres can have a dramatic effect, preventing dissolution in chemicals that are otherwise solvating the same tension-free cellulose. The effect of the tension was already well documented for cotton hairs in mercerization conditions [4]. It was shown that tension upon mercerization prevents the contractions, restricts the swelling and further increases the lustre, the tenacity and the Young's modulus and decreases the elongation at break. One of the main explanations given is the fact that the tension favours the alignment of the cellulose microfibrils within the fibre. Also, the tension upon mercerization was reported to increase much less the degree of disorder of cotton hairs than slack mercerization. Tension has thus an influence on structural changes that occur upon mercerization. However, no data are reported concerning the effect of the tension on the dissolution or the reactivity of cellulose fibres which are processes of primary importance for cellulose transformation.

The objectives of the present work are to investigate the role of the tension on the dissolution and the reactivity of cellulose fibres. This will be evaluated by studying the swelling and dissolution mechanisms of cotton hairs and regenerated cellulose fibres with and without tension. Thanks to these observations, the consequence of the tension upon derivatization will be evaluated by measuring the degree of acetylation of regenerated cellulose fibres using ${ }^{1} \mathrm{H}$ NMR spectroscopy and FTIR spectroscopy. Native cotton hairs and regenerated cellulose fibres were swollen and dissolved with and 
without tension in $N$-methylmorpholine- $N$-oxide (NMMO)-water solutions. Regenerated cellulose fibres were swollen with and without tension in mixtures of dimethyl sulfoxide (DMSO) and tetrabutylammonium fluoride trihydrate (TBAF) and derivatized to produce cellulose acetate.

\section{Materials and methods}

\subsection{Cellulose samples}

Cotton hairs, Gossypium barbadence, with mean diameter of $15 \mu \mathrm{m}$, were provided by INRA (France). Regenerated cellulose fibres (TENCEL®), Tencel fibres, with mean diameter of $11 \mu \mathrm{m}$ were provided by Lenzing AG (Austria). Another regenerated cellulose fibre (ALCERUß process), Alceru fibre, with a mean diameter of $12 \mu \mathrm{m}$ was provided by the Thuringische Institut für Textil und Kunstoff Forschung, Rudolstadt (TITK, Germany).

Cotton hairs are synthesized by nature and have a highly crystalline and very complex native wall structure with varying molecular orientation angle of cellulose chains (from 20 to $45^{\circ}$ to the fibre axis) depending of the considered layer $[1,4]$. The regenerated cellulose fibres used are Lyocell cellulose fibres, i.e. cellulose fibres obtained in a coagulation bath after a dry jet wet spinning of a solution of cellulose in a mixture of water and NMMO. Regenerated cellulose fibres are composed of highly oriented parallel cellulose fibrils connected by amorphous regions being also oriented $[5,6]$. Pores or voids are present in these fibres and were also shown to be elongated in the fibre direction [7,8]. AbuRous et al. $[9,10]$ showed that regenerated cellulose fibres contain only nanopores in the core of the fibre and a very porous skin layer. The structure of regenerated cellulose fibres is dependant of various parameters as the initial cellulose solution, the composition and the temperature of the aqueous regeneration bath and the spinning conditions [11,12]. 


\subsection{Solvents}

NMMO was provided by Sigma Aldrich in its anhydrous form. Mixtures of NMMO and water were prepared at $90^{\circ} \mathrm{C}$ and the water content was varied from 20 to $23 \% \mathrm{w} / \mathrm{w}$. NMMO-water mixtures are classical solvents used in the industry to dissolve cellulose. At $90^{\circ} \mathrm{C}$, the mixture is a solvent when the water content is above about $13 \%$ and below about $25 \%$, depending on the physical characteristics and provenance of the cellulose used. The behaviour of cotton hairs in NMMO-water mixtures has been reported to depend on water concentration $[13,14]$ : fast dissolution by fragmentation below $17 \%$ water, large swelling by ballooning, then dissolution between 19 to $23.5 \%$ water, large swelling by ballooning, but no complete dissolution between 25 to $30 \%$ water, homogeneous swelling and no dissolution between 35 to $40 \%$ water, and very low swelling above $40 \%$ water. In order to decrease dissolution kinetics (i.e. avoiding a fast disintegration of the fibres), we will use in this study water concentration in the NMMO-water solvent such as being able to dissolve regenerated cellulose fibres after a large swelling or cellulose cotton hairs after the production of balloons.

DMSO, TBAF, acetic anhydride, pyridine and 4-(dimethylamino)pyridine (DMAP) from Fluka were used as received. Köhler and Heinze [15] and Ass et al. [16] demonstrated that mixtures of DMSO/TBAF are good media for homogeneous derivatization reactions of cellulose under lab scale conditions.

\subsection{Experimental protocols}

Swelling, dissolution and derivatization trials were performed several times by different experimenters in order to check the reproducibility and the accuracy of the results.

\subsubsection{Swelling and dissolution in NMMO-water}

Experiments were performed by mixing the fibres and the solvent in a container made of two glass plates separated with double-sided tape. The solvent, previously heated at $90^{\circ} \mathrm{C}$ and contained in a pipette, was introduced by capillary forces between the two plates (Figure 1a), placed on a hot stage 
with a controlled temperature. No agitation was applied to the mixture. To understand the influence of the fibre tension and the accessibility to the lumen for cotton hairs on the swelling and dissolution mechanisms, four configurations of experiments were tested (Figure 1b). Considering the dimension of the fibres and the experimental assembly, it is not possible to measure the initial tension applied to the fibres. The four configurations were defined as follows: (i) no tension: fibres were fixed with a long, loose length between the two blocking points, (ii) low tension: fibres were fixed so that the contraction upon swelling leads to a low tension of the fibre, (iii) high tension: fibres were put under gentle tension between the two points of silicone grease. Tension was increased due to swelling. The set-up used to place fibres under axial tension has two side effects. It is preventing fibres to rotate when swelling and it is not allowing chemicals to penetrate into the lumen by the end of the cotton hairs. (iv) no tension but access of solvent to lumen: an access to the lumen was allowed with fibres free at one end.

\subsubsection{Swelling and dissolution in DMSO/TBAF}

A study of swelling and dissolution mechanisms with and without tension in DMSO/TBAF of isolated fibres was carried out at $60^{\circ} \mathrm{C}$ in a hot stage in a container made out of two glass plates. The solvent was introduced by capillary forces between the glass plates using a pipette. The preparation of the solvent was done by adding the quantity of TBAF to the DMSO solution in order to obtain mixtures of DMSO with up to $14 \%$ TBAF (w/w). Fibres free of tension were attached to one or two extremities and were free to move in the solvent. For inducing a tension, the fibre was attached to one extremity, stretched and then attached to the other extremity as was described above for experiments in NMMOwater.

\subsubsection{Tensile stress control}

As mentioned above, the magnitude of the tensile stress was not quantitatively controlled due to the experimental set-up. In the case called "high tension", the fibres were placed straight between the two blocking points (silicon grease, adhesive tape or clamps depending on the experiments). Since upon 
swelling, the fibres are contracting, this brings a tension which magnitude was not possible to measure, but in some cases, was high enough to break the fibres. As will be seen, the knowledge of the stress magnitude is not of primary importance for the interpretation of the results.

\subsubsection{Optical microscopy observations}

The swelling and dissolution mechanisms of isolated cotton hairs and regenerated cellulose fibres were observed by optical microscopy with a Metallux 3 (Leitz) equipped with a Linkam TMS 91 hot stage. The samples were investigated in transmission mode, at $90^{\circ} \mathrm{C}$ and $60^{\circ} \mathrm{C}$ for NMMO-water and DMSO/TBAF respectively. To obtain high resolution pictures, the microscope was equipped with a high resolution numerical reflex camera $(3000 * 2000$ pixels) CANON D100 (Figure 3) and a high resolution 3-CCD camera (1360*1024 pixels) JVC KY-F75U (Figure 4 and 5). In order to calculate the swelling ratio $=\frac{D}{D_{i}}$, the diameter of the fibre was measured before $\left(\mathrm{D}_{\mathrm{i}}\right)$ and after swelling (D).

\subsubsection{Derivatization procedure}

$500 \mathrm{mg}$ of Alceru fibres were first swollen in a solution composed of $15 \mathrm{ml}$ DMSO and $0.86 \mathrm{~g}$ TBAF $(\mathrm{DMSO} / 5 \% \mathrm{TBAF} \mathrm{w} / \mathrm{w})$. The swelling was carried out for 30 minutes at $60^{\circ} \mathrm{C}$ using the configuration of Figure 2a. The same procedure was followed for the fibres under tension using for easier handling an amount of $300 \mathrm{mg}$ of fibres with the same concentration (DMSO/5\% TBAF w/w), with the configuration of Figure 2b. Acetylation procedure for all fibres (with and without tension) was carried out as follows: the fibres swollen in the mixture of DMSO/TBAF were allowed to interact for 3 and 5 hours at $60^{\circ} \mathrm{C}$ (oil bath) with $5 \mathrm{~mol}$ acetic anhydride per mol anhydroglucose unit (AGU). The reaction was carried out in presence of $5 \mathrm{~mol}$ pyridine/AGU under magnetic stirring. The samples were then put in $300 \mathrm{ml}$ methanol, isolated by filtration, and washed three times with $250 \mathrm{ml}$ methanol. The resulting products were dried for two days in vacuum at $60^{\circ} \mathrm{C}$. 


\subsubsection{Perpropionylation}

Two different procedures were used for the fibres derived with or without tension. This is due to the large difference in the degree of substitution (DS) obtained with and without tension, as will be seen and discussed in the following parts of the paper. In both cases, it was checked that all the OH groups were fully substituted after the end of the protocols. For the perpropionylation of acetylated fibres without tension, $0.2 \mathrm{~g}$ of fibres were allowed to interact with $5 \mathrm{ml}$ pyridine and $5 \mathrm{ml}$ propionic anhydride in presence of $0.2 \mathrm{~g}$ of DMAP used as catalyst. The reaction was carried out for 24 hours at $80^{\circ} \mathrm{C}$, under magnetic stirring. The perpropionylation of the fibres acetylated under tension was carried out using 5 $\mathrm{ml}$ propionic anhydride and a mixture of $5 \mathrm{ml}$ DMSO with $0,54 \mathrm{~g}$ TBAF (DMSO/5\% TBAF w/w). The reaction was carried out for 1 hour at $80^{\circ} \mathrm{C}$. The resulting products were precipitated in a mixture of 200 $\mathrm{ml}$ methanol and water $(30 \%+70 \% \mathrm{w} / \mathrm{w})$ and washed three times in $150 \mathrm{ml}$ methanol + water (same concentrations as before). The resulting products were dried for 2 days in vacuum at $60^{\circ} \mathrm{C}$. The DS of the perpropionylated fibres were calculated according to equation 1 [17].

$$
D S_{A c y l}=3-\frac{7 \cdot I_{H, \text { propionyl }}}{3 \cdot I_{H, A G U}}
$$

where $I_{H, \text { propionyl }}$ is the peak integral of methyl protons moieties and $I_{H, A G U}$ is the peak integral of protons of the AGU.

\subsection{7. ${ }^{1} H$ NMR and FTIR measurements}

${ }^{1} \mathrm{H}$ NMR spectra were measured in deuterated chloroform $\left(\mathrm{CDCl}_{3}\right)$ and DMSO- $\mathrm{d}_{6}-50 \mathrm{mg} / \mathrm{mL}$ with a Bruker Advance 250 spectrometer running at $250 \mathrm{MHz}$ at room temperature. Sixteen scans were accumulated. FTIR spectra were recorded on Nicolet Avatar 370 DTGS spectrometer using both ATR and $\mathrm{KBr}$ techniques. 


\section{Experimental results}

\subsection{Influence of the tension on the swelling and dissolution mechanisms}

\subsubsection{Swelling and dissolution without tension in NMMO-water}

NMMO-water mixtures with water concentrations between 20 to $23 \%$ are slowly dissolving cotton hairs through a ballooning mechanism. During swelling, cotton hairs undergo a large rotation and an axial contraction increasing with swelling [18]. The breaking of the primary wall involved by the swelling and the dissolution of the inside of the fibre leads to successive highly swollen regions (the location of balloons) in between regions of the hair which are keeping about the original hair diameter (unswollen regions). Balloons are formed by a membrane, surrounded by helices from the primary wall, and composed of older deposited secondary wall layers (S1 layer) enclosing dissolved cellulose from S2 layer. The sequence of dissolution is the following: first the inside of the fibre (S2 layer) by fragmentation, then the S1 wall, then the unswollen regions and remaining of the primary wall [14]. For the cotton hairs used, balloons reach a maximum swelling ratio of 5.3 in NMMO-water 20 to $23 \%$ (Figure 3a). Tencel fibres swell and then dissolve in NMMO-water mixtures with water concentration at $20 \%$ (Figure 4a). The maximum swelling ratio can reach 9.5 and the swollen fibre aspect is very translucent and difficult to visualize by optical microscopy. Tencel fibres are also contracting upon swelling, but they are not rotating. The fact that the Tencel fibres show higher swelling as compared to cotton hairs can be explained by the longitudinal orientation of their cellulose chains which do not impede the radial swelling.

\subsubsection{Swelling and dissolution under low tension in NMMO-water}

When placed in the water concentration range of 20 to $23 \%$, loosely attached cotton hairs swell homogeneously and contract. This induces a low tension state with a maximum swelling ratio of about 4.8 (Figure 3b), similar to the swelling of the balloons (5.3) observed without tension right before their 
dissolution. Swelling starts at localized places along the fibre but contrary to the tension-free case where the rolling of the primary wall limits its extension by forming unswollen regions $[13,14,18]$, swelling extends along large dimensions along the hair until all the swelling regions merge. In marked contrast with the case without tension, hairs under low tension have no balloon and they are not dissolving: they are staying homogeneously swollen. In this homogeneous swollen state, the fibre is thus only composed of older deposited secondary wall layers (S1 layer) enclosing dissolved cellulose from S2 layer. In the case of Tencel fibres, for a water concentration of $20 \%$, fibres swell homogeneously and contract to reach a maximum swelling of about 5.2 (Figure 4b). This swelling depends of the initial length of fibre over the length between the two blocking points and can vary from 4.5 to 5.5 . The main observation is that regenerated cellulose fibres and cotton hairs stay in this large swollen state and do not dissolve, contrary to the case with no axial tension. Without tension, the same swelling ratio would allow regenerated fibres and cotton hairs to dissolve.

This is the first important result showing that what is controlling dissolution is not the degree of swelling, since at the same degree of swelling, fibres under tension are not dissolving while fibres without tension are.

\subsubsection{Swelling and dissolution under high tension in NMMO-water}

Between 20 and $23 \%$ of water, cotton hairs swell homogeneously and do not dissolve, but their swelling decreases with increasing tension strength (Figure 3c). A very interesting point is that if tension is released suddenly as it occurs sometimes when the cotton hairs break due to a too high tension generated by the contraction, cotton hairs are dissolving immediately. The same phenomena are observed with Tencel fibres.

This is the second important results showing that under tension, when the solvent is present around cellulose chains, it is activated to solvate the chains only when stress is released. 


\subsubsection{Accessibility to the lumen in NMMO-water}

Since the set-up leading to placing cotton hairs under axial tension was preventing access of solvents to the lumen, we checked that cotton hairs without tension and without access to the lumen were still showing the same swelling and dissolution mechanisms as free hairs. Lack of lumen access is not the reason for the above-observed phenomena when cotton hairs are placed under tension.

The above results thus show that tension is preventing cotton hairs and regenerated cellulose fibres to dissolve in NMMO-water mixtures that would dissolve the same cellulose fibre tension-free. In the next paragraph, we will check the influence of the tension on the derivatization of regenerated cellulose fibres.

\subsection{Influence of the tension on the derivatization}

\subsubsection{Preliminary study of swelling and dissolution in DMSO/TBAF}

Alceru fibres with or without tension show a low swelling ratio (lower than 1.5) in pure DMSO. When the fibres without tension are dipped in $1 \%$ to $5 \%$ TBAF in DMSO, a much larger swelling occurs, starting with formation of sorts of rings localized on different zones of the fibre and finishing with a homogeneous swelling. With the fibres under tension, swelling ratio is about the same as without tension at $1 \%$ of TBAF in DMSO but much lower under tension at $5 \%$ concentration of TBAF (Table 1). Since at a concentration of $5 \%$ of TBAF in DMSO, the maximum swelling occurs (above $7 \%$ of TBAF, fibres are dissolving), this concentration was used in the derivatization experiments

\subsubsection{Derivatization of regenerated cellulose fibres}

Acetylation of Alceru fibres was performed in DMSO containing 5\% TBAF with and without tension. Cellulose fibres without tension are first reacting heterogeneously, and then turned to homogeneous mixtures as soon as the degree of acetylation reaches a critical point where the cellulose acetate is soluble in the reaction medium. In contrast, when the acetylation was carried out under 
tension, the degree of substitution is limited and is below this critical point, and the reaction stays heterogeneous. The influence of tension is thus to limit the extent of derivatization, which is blocked at a low degree of substitution. In contrast, without tension, the degree of substitution is increased up to the dissolution point where the reaction turns homogeneous, increasing even further the degree of substitution.

After acetylation and isolation of the products, the presence of acetyl groups was checked by FTIR spectroscopy. Typical spectra obtained for fibres after acetylation with and without tension are given in Figure 5. Both fibres show the typical vibrations of the ester moiety at about $v=1730 \mathrm{~cm}^{-1}$, indicating that hydroxyl groups were esterified. Comparing the intensities of the $\mathrm{OH}$ - and ester signals for the differently acetylated fibres shows clearly that the fibres derivatized under tension possess a lower DS. The value of the DS could be determined by ${ }^{1} \mathrm{H}$ NMR spectroscopy.

${ }^{1} \mathrm{H}$ NMR spectroscopy of perpropionylated cellulose acetate is a useful tool to determine the DS of the samples. After perpropionylation of the remaining $\mathrm{OH}$ groups, the chemical shifts of the acetyl- and the propionyl moieties in the range 1.00 to $2.5 \mathrm{ppm}$ were detected. The signals of the AGU are located between 3.3 and $5.25 \mathrm{ppm}$. A typical spectrum obtained after perpropionylation of the acetylated fibres without tension is shown on Figure 6. It is in good agreement with previous studies [17]. The DS was calculated according to equation 1 . The DS values of the fibres as a function of the reaction conditions (with or without tension, reaction time) are given in Table 2.

The samples treated without tension have DS in the range of 1.1-1.3 (table 2) increasing with the reaction time (Figure 7). These DS values are lower compared to samples that are synthesized with native cellulose. The derivatization of native cellulose fibres without tension from the polymer dissolved in a mixture of DMSO/5.7\% TBAF was shown to lead to DS values between 1.5 and 2.3 at a molar ratio of 13 mol of acetic anhydride per mol AGU [15]. With tension, the DS values are much smaller, in the range of $0.2-0.5$ (Figure 7). As can be seen on Table 2, the resulting products are highly soluble in both pyridine and DMSO when no tension was applied during the reaction. In contrast, fibres that were reacting under tension are not soluble. 


\section{Discussion}

Dissolution of a semi-crystalline polymer in a solvent proceeds classically through the local swelling of the polymer at the polymer-solvent interface followed by disentanglement and diffusion into the solvent [19]. There is no need for the whole polymer part to be accessible to the solvent prior to dissolution. Due to the difficulties for dissolving or chemically treating cellulose, it has always been postulated, and taken for granted, that the whole cellulose part must be first brought accessible to the solvent by methods like (i) opening of the pores present in native or regenerated cellulose, (ii) swelling non-crystalline regions, (iii) breakage of the crystalline areas, (iv) weakening the hydrogen-bond array by chemical or physical treatments or (v) decreasing molecular weight by chemical or enzymatic treatments. It is implicitly assumed that the classical mechanisms of polymer dissolution are not applicable to cellulose. The main result of this work is the absence of dissolution and a large decrease of chemical reactivity when cellulose fibres are put under tension and prevented to contract.

In NMMO-water, the swelling reached under low tension is of similar magnitude as the swelling of balloons seen during the dissolution of cotton hairs without tension. Similar swellings are also observed with and without tension for regenerated cellulose fibres. This shows that the opening of pores or the need for the cellulose chains to be close to the solvent are not the major reasons why cellulose is not fully dissolving in this solvent. The fact that a swollen cotton hair or a regenerated fibre under high tension is immediately dissolving when the fibre is breaking (breakage due to the tension stress generated by the swelling-induced contraction) shows that the solvent was present around chains prior to cotton hair breakage and was able to disrupt then the whole structure, solvating chains or chain aggregates. In DMSO/TBAF, swelling of fibres under tension is large (swelling ratio of 8) but smaller than without tension (swelling ratio of 14). However, a swelling ratio of 8 is still important and implies that a lot of swelling agent is present in the fibre (,the initial diameter is increased by a factor 8 and the total volume of the fibre by a factor 64 considering that the length of the fibre stays constant during swelling). Whatever is the precise figure of the increase of volume, its large value should allow the penetration of reactants in a large proportion, not very different from the case of swelling without 
tension. The accessibility of chains to solvent is thus not the full story for explaining cellulose dissolution and treatments.

The reactivity with and without tension is very different when starting from the same highly swollen state of the cellulose chains. Without tension, the fibre is highly swollen and the derivatization proceed up to DS values that are high enough to dissolve the derivatized cellulose, increasing further the degree of derivatization. Under tension, derivatization is not very efficient and is kept at such low values that no dissolution occurs upon derivatization. The final result of DS showing a large difference between the two cases (DS values being 0.2-0.5 under tension and 1.1-1.3 without tension) is biased by the fact that in the no tension case, the reaction has a transition towards a homogeneous derivatization at the end of the process. The step of great interest is when both reactions are in the heterogeneous state (the fibre is swollen but not dissolved), i.e. at the beginning of the chemical reactions. When the fibre is under tension, the acetylation is strongly inhibited, blocked at a DS of $0.2-0.5$, while the DS is increasing much above this with no tension. It shows that to keep the fibres, and by extension the microfibrils and the cellulose chains, under tension is strongly limiting the efficiency, despite the swelling is very large and thus the fibres accessible to the chemicals. The reactivity of cellulose and hence the DS obtained can be limited by several factors. (i) Aggregation of chains or at least strong interactions in the swollen state is one serious issue. It can be assumed that the higher the swelling is, the more separated the chains are, and thus the more reactive they are. This cannot explain the difference between the cases under and without tension since the swelling ratio is already very high in the tension case. (ii) Change of structure as a result of the derivatization (acetylation) may occur sometimes leading to deswelling and in case of a dissolved polymer to insolubility and hence precipitation. The chemical reaction being similar with or without tension, this cannot explain the difference. (iii) Also a change in the equilibrium of the reaction can be ruled out since the chemical reactions are similar with and without tension. Thus, typical factors limiting reactivity cannot be easily used for explaining the significant difference in reagent reactivity and in the conversion of the cellulose fibres without tension and under tension. The low DS values of the fibres treated under tension could also be due to an uneven distribution of substitution inside the 
fibre. It could be supposed that under tension, for an unknown reason, the reaction is only possible within a small core thickness, giving a low figure when the measurement of the substitution degree is averaged over the whole fibre. One way to check this was to see whether acetylated fibres under tension have a swelling behaviour that would show that the outside of the fibre behaves differently compared with the inside. The results obtained indicate that acetylated fibres synthesized under tension possess a homogeneous swelling behavior, suggesting that the acetyl moieties are distributed homogeneously within the fibre volume. In addition, if only the outside of fibres would be highly substituted, this part should be soluble in DMSO containing 5\% TBAF, which is not the case. We can thus conclude that under tension, the fibres are not much substituted and rather homogeneously inside the whole fibre. Tension is inhibiting the reactivity of the reacting agent despite it has access to the cellulose chains, considering the high swelling ratio reached.

The fact that both cotton hairs and well oriented regenerated cellulose fibres are showing dissolution and reactivity inhibitions under tension indicates that the detailed structural morphology of the cellulose fibre is not involved. We will postulate here a simple explanation able to describe the observed phenomena. The common point is the fact that cellulose chains are oriented and interlinked into strong intermolecular hydrogen bond arrays [1,2]. Dissolution or chemical reaction impose that the reacting or solvating molecule approaches the cellulose chains and couples to it, inducing local conformational movements that depends on the reagent involves and to which site of the AGU it binds with. With few exceptions like crankshaft motions, local movements impose the chain to rearrange its conformation along its axis. The lack of reactivity under tension, despite having reagent accessibility, shows that the local conformations necessary for solvatation or chemical reaction of the chains are prevented. This can be understood if we consider that cellulose chains are in straight, parallel conformation even in the noncrystalline regions in natural cotton hairs [20] and regenerated cellulose fibres [5,6] and that an hydrogen bond array is still linking all chains and microfibrils together in the entire sample, even with the large swelling involved. It implies that not all the intra and inter-molecular hydrogen bonds are broken. When the length of the fibre is fixed by tension, local molecular movements needed for the 
solvatation and for chemical reaction of the chains are inhibited. Without being able to perform these movements, chains are not dissolving and have difficulties to react. In contrast, when tension is not applied or released, the molecular movements are favoured and able to disrupt the remaining hydrogen bonds. The need of spatial mobility, at least the possibility that specific conformations are accessible, could thus be an important factor to be taken into account for cellulose dissolution and reactivity.

\section{Conclusions}

To be able to dissolve or to be chemically treated, we are postulating that cellulose chains must be allowed to perform local conformational movements requiring long range chain mobility, not possible when the chain is blocked within its hydrogen bond array. It is not enough for the chains to be close to the reagent or to be not crystalline. This result is suggesting that activation must concentrate on finding better ways to break the hydrogen bond array and prevent its reformation. Further work focusing on polymer conformation, interactions with reagent and movement (for example by NMR) under tension are needed to better assess the physical phenomena underlining the observed results. We believe that studying dissolution, chemical reactions or any other imposed constraint on cellulose (and possibly on other polymers having strong hydrogen bond arrays) can be an interesting new way to better understand cellulose molecular dynamics and chain physics and to improve existing cellulose treatments for dissolution.

Acknowledgements. PN and NLM thank J. Engelhardt, K. Jardeby, T. Hjerde, H. Harms, H. Höfte, M. Pierre, C. Pannetier and C. Schrempf for discussions and Borregaard, Dow Wolff Cellulosics GmbH, Lenzing AG and Spontex for their technical and financial support. PN, TH and MS thank the Thuringische Institut für Textil und Kunstoff Forschung for providing regenerated cellulose fibres. MS thank the support of EC Polysaccharide "EPNOE" project for her stay in Jena. 


\section{References}

1. Krässig HA. In: Huglin MB editor. Cellulose structure, accessibility and reactivity, Polymer Monographs, vol. 11. Amsterdam: Gordon and Breach Science Publishers, 1993 (chapter 2).

2. Klemm D, Philipp B, Heinze T, Heinze U. Wagenknecht, W. Comprehensive cellulose chemistry, vol. 1. Weinheim: Wiley-VCH, 1998 (chapter 2).

3. Isogai A, Atalla RH. Cellulose 1998;5(4):309-319.

4. Warwicker JO, Jeffries R, Colbran RL, Robinson RN. A review of the literature on the effect of caustic soda and other swelling agents on the fine structure of cotton, Shirley Institute Pamphlet, vol. 93.

England: St Ann's Press, 1966. pp. 25-70

5. Lenz J, Schurz J, Wrentschur E. Colloid Polym Sci 1993;271(5):460-468.

6. Schurz J. Lenzinger Ber 1994;74:37-40.

7. Crawshaw J, Vickers ME, Briggs NP, Heenan RK, Cameron RE. Polymer 2000;41(5):1873-1881.

8. Crawshaw J, Cameron RE. Polymer 2000;41(12):4691-4698.

9. Abu-Rous M, Ingolic E, Schuster KC. Cellulose 2006;13(4):411-419.

10. Abu-Rous M, Varga K, Bechtold T, Schuster KC. J Appl Polym Sci 2007;106(3):2083-2091.

11. Biganska O, Navard P. Cellulose 2009;16(2):179-188.

12. Schuster KC, Rohrer C, Eichinger D, Schmidtbauer J, Aldred P, Firgo H. Environmentally friendly lyocell fibres. In: Wallenberger FT, Weston NE, Editors. Natural Fibres, Plastics and Composites. Boston, Dordrecht, New York, London: Kluwer Academic Publishers, 2003. pp. 123-146.

13. Cuissinat C, Navard P. Macromol Symp 2006;244(1):1-18.

14. Le Moigne N, Montes E, Pannetier C, Höfte H, Navard P. Macromol Symp 2008;262(1):65-71.

15. Köhler S, Heinze T. Macromol Biosci 2007;7(3):307-314.

16. Ass BAP, Frollini E, Heinze T. Macromol Biosci 2004;4(11):1008-1013.

17. Heinze T, Liebert T, Koschella A. Esterification of polysaccharides. Berlin, Heidelberg, New York: Springer, 2006 (chapter 8). 
18. Le Moigne N, Bikard J, Navard P. Rotation and contraction of native and regenerated cellulose fibres upon swelling and dissolution: the role of morphological and stress unbalances, submitted to Cellulose

19. Miller-chou BA, Koenig JL. Prog Polym Sci 2003;28(8):1223-1270.

20. O'Sullivan AC. Cellulose 1997;4(3):173-207. 


\section{Graphical abstract}

Restricted dissolution and derivatization capacities of cellulose fibres under uniaxial elongational stress

Nicolas Le Moigne, Monica Spinu, Thomas Heinze and Patrick Navard
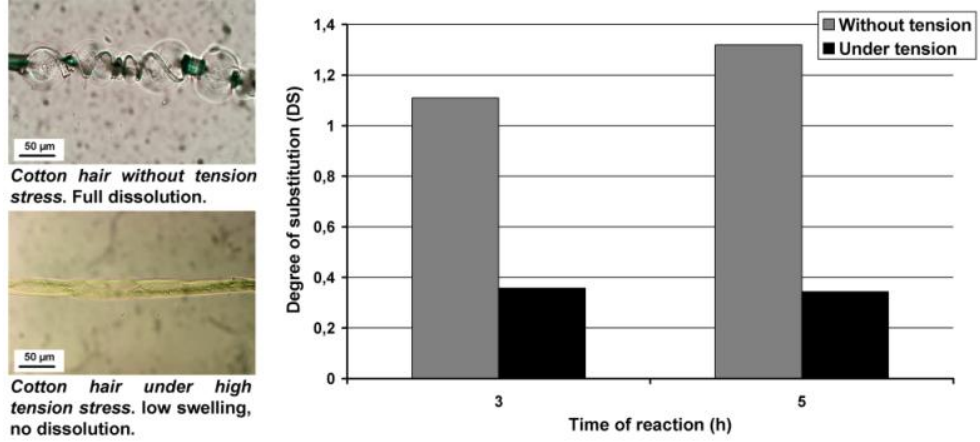


\begin{tabular}{ccc}
\hline $\begin{array}{c}\text { TBAF } \\
\text { concentration }\end{array}$ & $\begin{array}{c}\text { Swelling ratio } \\
\text { without tension }\end{array}$ & $\begin{array}{c}\text { Swelling ratio } \\
\text { with tension }\end{array}$ \\
\hline $0 \%$ & $<1.5$ & $<1.5$ \\
$1 \%$ & 9 & 8.7 \\
$5 \%$ & 14 & 8
\end{tabular}

Table 1. Swelling ratio of Alceru fibres in $\mathrm{DMSO} / \mathrm{x} \% \mathrm{TBAF}$ at $60^{\circ} \mathrm{C}$.

\begin{tabular}{ccccc}
\hline \multirow{2}{*}{ Tension } & Time $(\mathrm{h})$ & DS & \multicolumn{2}{c}{ Solubility } \\
\cline { 4 - 5 } & & & Pyridine & DMSO \\
\hline no & 3 & 1.11 & yes & yes \\
yes & 3 & 0.48 & no & no \\
yes & 3 & 0.33 & no & no \\
yes & 3 & 0.26 & no & no \\
& & & & \\
no & 5 & 1.32 & yes & yes \\
yes & 5 & 0.43 & no & no \\
yes & 5 & 0.38 & no & no \\
yes & 5 & 0.32 & no & no \\
yes & 5 & 0.24 & no & no
\end{tabular}

Table 2. Reactions conditions and DS obtained for the derivatized fibres. The reactions were carried out using a solution of DMSO/5\% TBAF (w/w). The molar ratio of acetic anhydride per anhydrogluycose unit (AGU) was 1:5 and the temperature of reaction was $60^{\circ} \mathrm{C}$. 
a
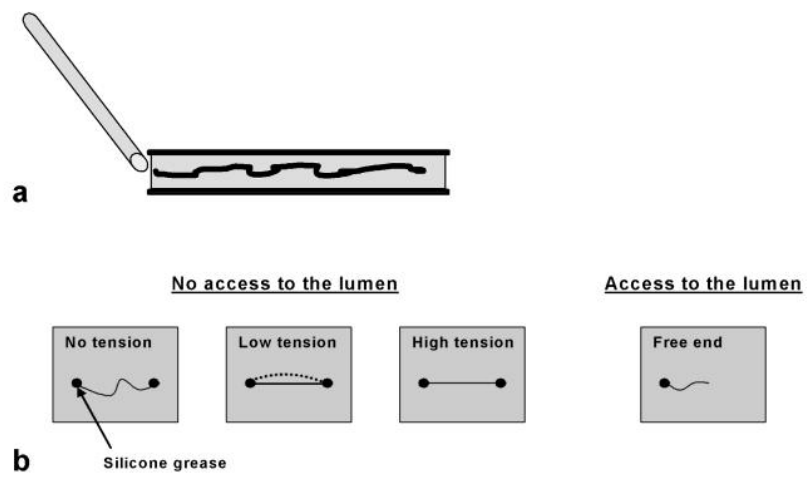

Figure 1. Experimental protocol and assembly for studying swelling and dissolution behavior of cellulose fibres in NMMO-water at $90^{\circ} \mathrm{C}$. (a), The solvent is injected by capillarity between the two glass plates. (b), Four configurations of experiments: (i) no tension, (ii) under low tension (the fibre loosely attached reaches a low tension state due to the contraction during swelling), (iii) under higher tension, and (iv) free end with an access to the lumen in the case of cotton hairs.

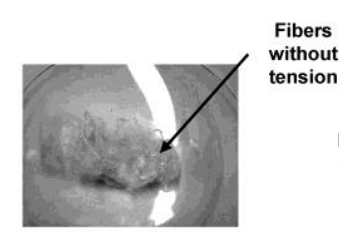

a

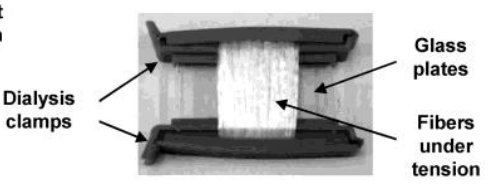

b

Figure 2. Experimental assembly for derivatization experiments: (a) arrangement for derivatizating fibres without tension (fibres were simply placed in a random manner in the container with the chemical reagents) and (b) with tension (fibres were attached between two dialysis clamps in order to stretch them). 


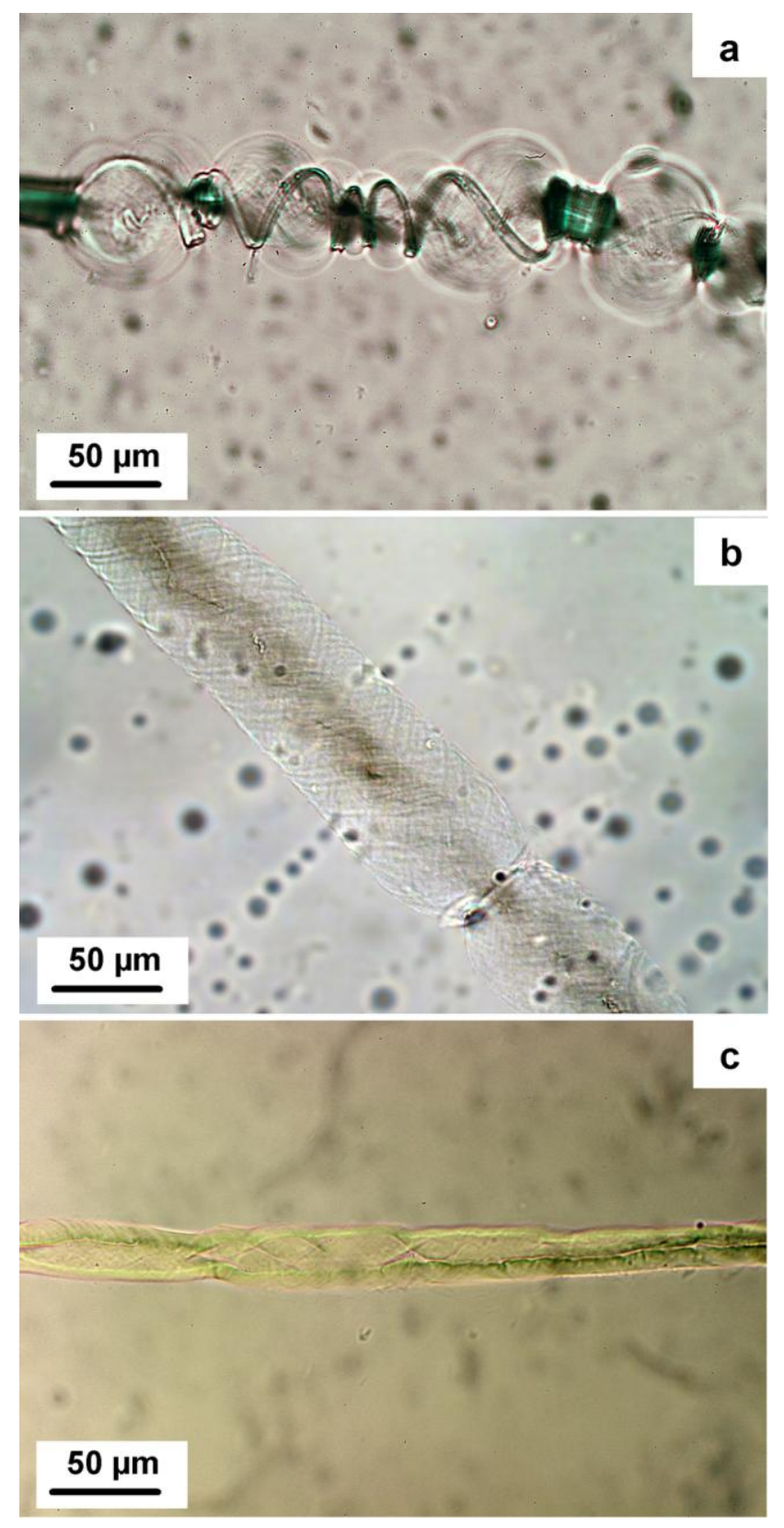

Figure 3. NMMO-water: swollen cotton hairs under varied conditions of tension. (a), Swelling by ballooning (ratio 5.3) right before the dissolution, no tension was applied to the fibre. (b), Large homogeneous swelling (ratio 4.8) and no dissolution, the fibre is under low tension. (c), Low homogeneous swelling (ratio 2) and no dissolution, a higher tension was applied to the fibre. 


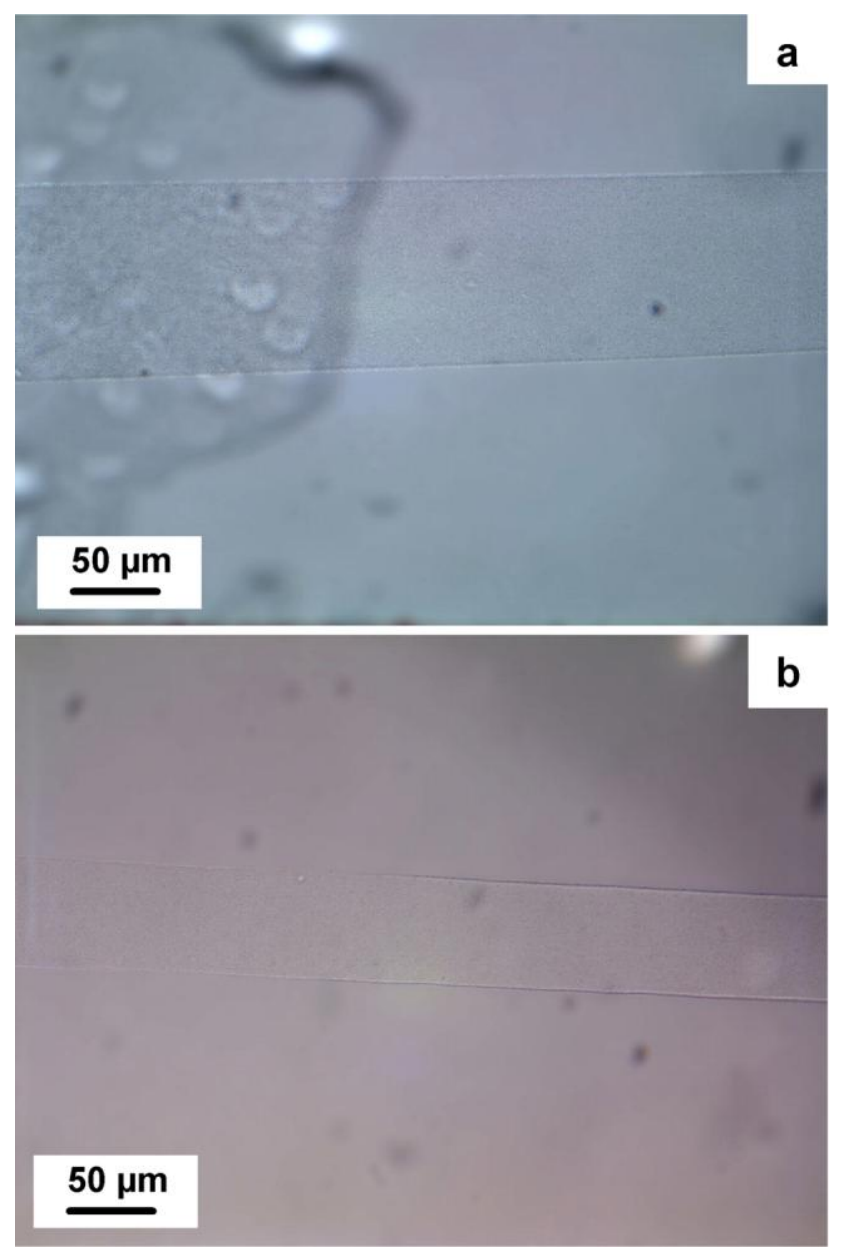

Figure 4. NMMO-water: swollen Tencel fibres under varied conditions of tension. (a) Very large homogeneous swelling (ratio 9.5) right before the dissolution, no tension was applied to the fibre. (b), Lower homogeneous swelling (ratio 5.2), the fibre is under low tension. 


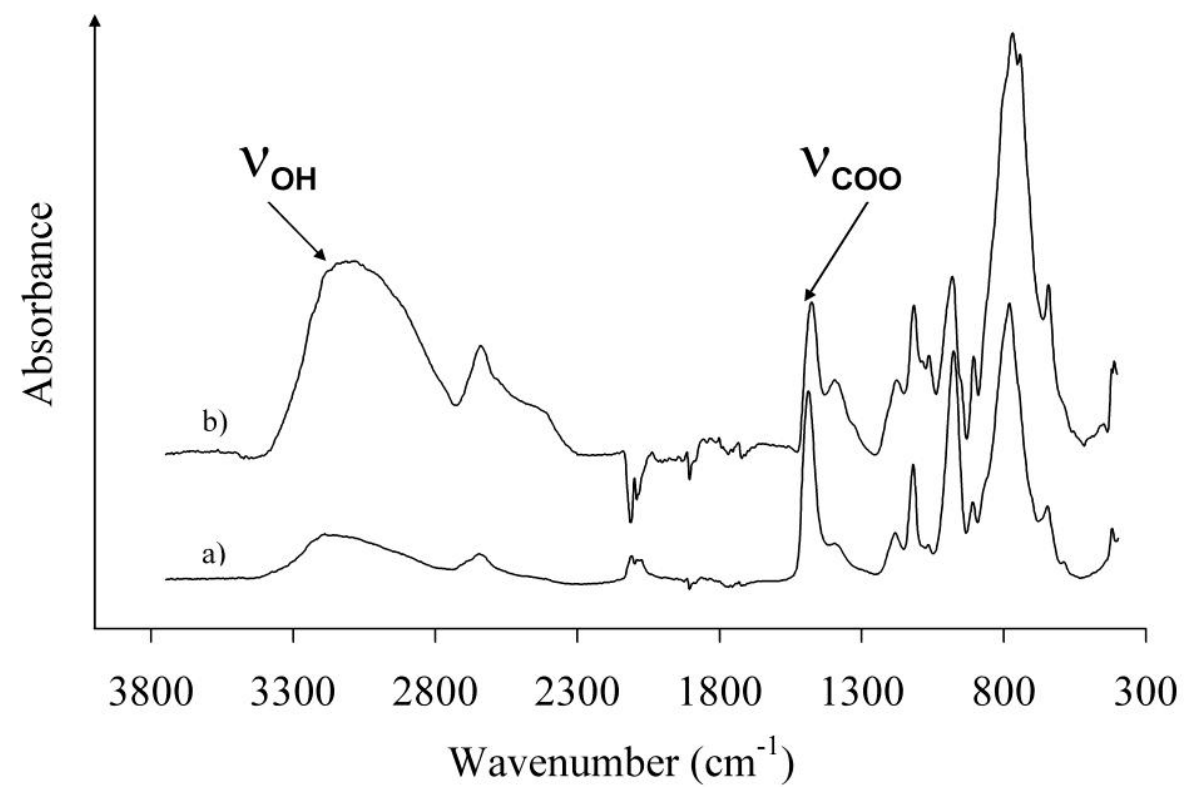

Figure 5. Example of FTIR spectra of acetylated Alceru fibres: a) without tension; b) with tension. 


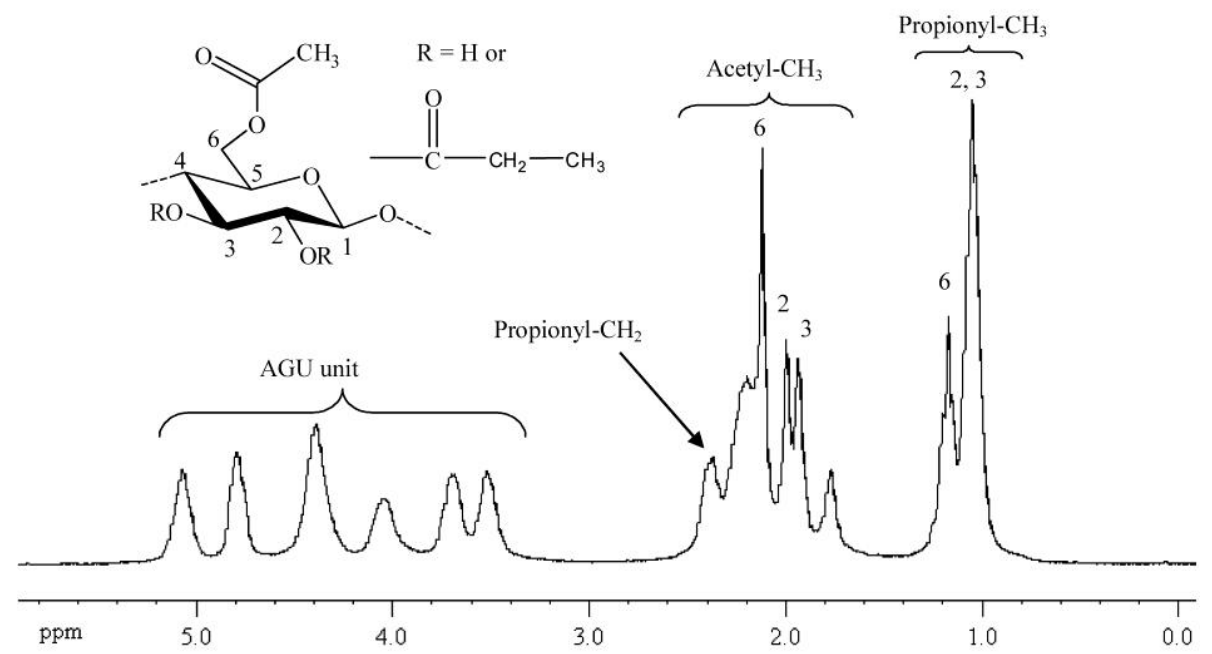

Figure 6. ${ }^{1} \mathrm{H}-\mathrm{NMR}$ spectra of the perpropionylated samples without tension, solvent is deuterated chloroform $\left(\mathrm{CDCl}_{3}\right)$.

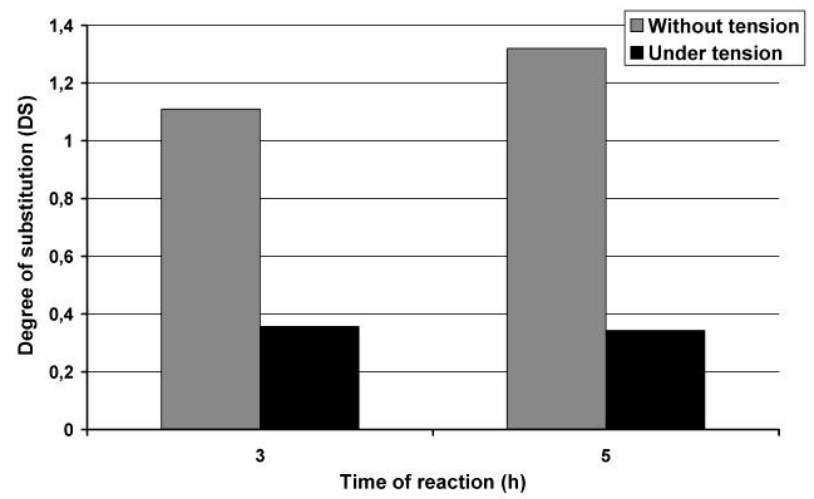

Figure 7. Evolution of the DS with and without tension versus the time of reaction. 